Author: SKACHKOV Vladimir Mikchaylovich, PhD, Chemistry, researcher of the Federal State government-financed research institution Institute of Solid State Chemistry of the Ural Branch of RAS; Pervomaiskaya str., 91, Ekaterinburg, Russia, 620990, e-mail: vms@weburg.me;

Author: YATSENKO Sergey Pavlovich, Doctor of Chemistry, Professor, honored worker of science and technology of the Russian Federation, principal researcher of the Federal State governmentfinanced research institution Institute of Solid State Chemistry of the Ural Branch of RAS; Pervomaiskaya str., 91, Ekaterinburg, Russia, 620990, e-mail: yatsenko@ihim.uran.ru

\title{
MODIFICATION OF ALUMINIUM ALLOYS WITH RARE METALS - THE BASIS FOR ADVANCED MATERIALS IN CONSTRUCTION AND TRANSPORT
}

\section{Extended Abstract:}

The method of process powder injection into aluminum melt shows much promise. Scandium is injected by the high-temperature exchange reaction between the salt melt and aluminum. The best salt compositions were selected. The results of the process are considered to depend on the initial salts. A series of fusions was performed under production conditions at the Kamensk-Uralskii metallurgical plant. It was shown that the injection method for production of aluminoscandium master alloys is technologically feasible. To protect intellectual property of authors, employees of the Institute of Solid State Chemistry, Ural branch of RAS (Russia) a patent «Method of alloying of aluminium or alloys on its basis» RU № 2534182 of 27.11 .2014 was registered.

Key words: injection, aluminum, scandium, fluoride, chloride, salts, powder, alloy.

DOI: dx.doi.org/10.15828/2075-8545-2016-8-3-60-69

MACHINE-REAdABLE INFORMATION ON CC-LICENSES (HTML-CODE) IN METADATA OF THE PAPER

$<$ a rel="license" href="http://creativecommons.org/licenses/by/4.0/" ><img alt="Creative Commons License" style="borderwidth:0" src="https://i.creativecommons.org/1/by/4.0/88x31.png" $/></ \mathrm{a}><$ br $/><$ span xmlns:dct="http://purl.org/ $\mathrm{dc} /$ terms/" href="http://purl.org/dc/dcmitype/Text" property="dct:title" rel="dct:type" $>$ Modification of aluminium alloys with rare metals - the basis for advanced materials in construction and transport. $</$ span $>$ by $<$ a xmlns:cc="http:// creativecommons.org/ns\#" href="Nanotehnologii v stroitel'stve = Nanotechnologies in Construction. 2016, Vol. 8, no. 3, pp. 60-69." property="cc:attributionName" rel="cc:attributionURL">Skachkov V.M., Yatsenko S.P. $</$ a $>$ is licensed under a $<$ a rel="license" href="http://creativecommons.org/licenses/by/4.0/">Creative Commons Attribution 4.0 International

License $</ \mathrm{a}>$. $<$ br $/>$ Based on a work at $<$ a xmlns:dct="http://purl.org/dc/terms/" href=" http://nanobuild.ru/en_EN/ nanobuild-3-2016/" rel="dct:source"> http://nanobuild.ru/en_EN/nanobuild-3-2016/</a $>$. $<$ br $/>$ Permissions beyond the scope of this license may be available at <a xmlns:cc="http://creativecommons.org/ns\#" href="vms@weburg.me" rel="cc:morePermissions">vms@weburg.me $</ \mathrm{a}>$. 


\section{References:}

1. Mondolfo L.F. Struktura I svoystva aluminievih splavov [Structure and properties of aluminum alloys]. Moscow, Metallurgy, 1979. 640 p. (In Russian).

2. Yatsenko S.P., Pasechnik L.A., Skachkov V.M. Skandiy: poluchenie i primenenie [Scandium: synthesis and application]. Science and technology, 2015. No. 3 (15). P. 32-37. (In Russian).

3. Belov N.A., Alabin A.N., Eskin D.G. et. al. Optimization of Hardening of Al-Zr-Sc cast alloys J. Mater Sci. 2006. No. 41. P. 5890-5899.

4. Fuller Ch.B., Seidnan D.N., Dunand D.C. Mechanical properties of Al (Sc,Zr) alloys of ambient and elevated temperatures. Acta Materialia. 2003. V. 51. P. 48034814.

5. Harada Y., Dunand D.C. Microstructure of Al3Sc witu ternary transition-metal additions. Materials Scien. a. Engineering. 2002. V. A. 329-331. P. 686-695.

6. She. Jia, Y. Zman, Zh. Hu et. al. Experimental Study of Al-Zr-Y system phase equilbria of 773K. J. of Alloys and Compounds. 2010. V. 497. P. 118-120.

7. Rokhlin L.L., Bogar N.P., Dobatkina T.V. et al. Issledovaniy bogatoy aluminiem chasti diagrammy sostoianiy Al-Hf [The study is rich in aluminum parts of a phase diagram of Al-Hf]. Metals. 2009. No. 3. P. 93-98. (In Russian).

8. Filatov Y.A. Al-Mg-Sc-splavy dly svarnyh konstrukzii [Al-Mg-Sc-alloys for welded structures]. Technology of light alloys. 2004. No. 5. P. 13-19. (In Russian).

9. Kazantsev V.P., Beketov L.R., Kudryavskiy Y.P. et. al. Perspektivi izvlecheniy skandiy iz rastvorov podzemnogo vischelechivaniy mineralnogo syria [Prospects for the extraction of scandium from solutions of underground leaching of mineral raw materials]. Nonferrous metallurgy. 2009. No. 1. P. 37-41. (In Russian).

10. Chertovikov $V$. Problema v tom, chto vozim ne passajirov, a taru [The Problem is that we carry no passengers, and Tara]. Metals of Eurasia. 2007. No. 2. S. 28-29. (In Russian).

11. Khokhlova L.B., Kolobnev N.I., Mikhailov E.D. Aluminiylitievie splavi dly samoliotostroeniy [Aluminium-lithium alloys for aircraft industry]. Metallurgy. 2012. No. 5. P. 31-35. (In Russian).

12. Ovsyannikov B.V. Novii aluminievo-litievii splav sistemi Al-Cu-Mg-Li(Ag, Sc), prednaznachenii dly izgotovleniy tonkih listov I profilei [New aluminum-lithium alloy of $\mathrm{Al}-\mathrm{Cu}-\mathrm{Mg}-\mathrm{Li}(\mathrm{Ag}, \mathrm{Sc})$, intended for the manufacture of thin sheets and profiles]. Non-ferrous metals. 2014. No. 11. P. 90-94. (In Russian). 
13. Ovsyannikov B.V., Yatsenko S.P., Varchenia P.A. et. al. Poluchenie aluminii-skandievih splavov metodom injekzii tehnologicheskih porosckov v rasplav [Obtaining aluminium-scandium alloys by injection of technological powders to the melt]. Technology of metals. 2011. No. 5. P. 23-29. (In Russian).

14. Skachkov V.M., Yatsenko S.P. Poluchenie Sc-, Zr-, Hf-, Y-ligatur na osnove aluminiy metodom visokotemperaturnih obmennih reakzii v rasplavah solei [Preparation of the Sc-, Zr-, Hf-, Y-alloys based on aluminum using high-temperature exchange reactions in salt melts]. Non-ferrous metals. 2013. No. 12. P. 48-52. (In Russian).

15. Kablov E.N. Bez novih materialov - net buduschego [Without new materials - no future]. Metallurg. 2013. No. 12. P. 4-8. (In Russian).

Dear Colleagues!

THE REFERENCE TO THIS PAPER HAS THE FOLLOWING CITATION FORMAT:

Skachkov V.M., Yatsenko S.P. Modification of aluminium alloys with rare metals the basis for advanced materials in construction and transport. Nanotehnologii $\mathrm{v}$ stroitel'stve $=$ Nanotechnologies in Construction. 2016, Vol. 8, no. 3, pp. 60-69. DOI: dx.doi.org/10.15828/2075-8545-2016-8-3-60-69. (In Russian). 
Автор: СКАЧКОВ Владимир Михайлович, кандидат химических наук, научный сотрудник, Федеральное государственное бюджетное учреждение науки Институт химии твердого тела Уральского отделения РАН; ул. Первомайская, 91, г. Екатеринбург, Россия, 620990, e-mail:vms@weburg.me;

Автор: ЯЦЕНКО Сергей Павлович, доктор химических наук, профессор, заслуженный деятель науки и техники РФ, главный научный сотрудник, Федеральное государственное бюджетное учреждение науки Институт химии твердого тела Уральского отделения РАН;

ул. Первомайская, 91, г. Екатеринбург, Россия, 620990, e-mail: yatsenko@ihim.uran.ru

\section{МОДИФИЦИРОВАНИЕ АЛЮМИНИЕВЫХ СПЛАВОВ РЕДКИМИ МЕТАЛЛАМИ - ОСНОВА ПЕРСПЕКТИВНЫХ МАТЕРИАЛОВ В СТРОИТЕЛЬСТВЕ И ТРАНСПОРТЕ}

АННОТАЦИЯ К СТАТЬЕ (АВТОРСКОЕ РЕЗЮMЕ, РЕФЕРАТ):

Метод инжекции технологического порошка в расплав алюминия является перспективным. Введение скандия осуществляется высокотемпературной обменной реакцией солевого расплава и алюминия. Производился подбор наилучшего солевого состава. Обсуждены результаты процесса в зависимости от исходных солей. Проведен ряд плавок в производственных условиях на ОАО «Каменск-Уральском металлургическом заводе». Показана технологичность инжекционного метода получения алюмоскандиевых лигатур. С целью защиты интеллектуальной собственности авторами - сотрудниками ИХТТ УрО РАН (Россия) - был зарегистрирован патент РФ «Способ легирования алюминия или сплавов на его основе» // Патент РФ № 2534182 от 27.11.2014.

Ключевые слова: инжекция, алюминий, скандий, фторид, хлорид, соли, порошок, сплав.

DOI: dx.doi.org/10.15828/2075-8545-2016-8-3-60-69 
МАШИНОЧИТАЕМАЯ ИНФОРМАЦИЯ о СС-ЛИЦЕНЗИИ В МЕТАДАННЫХ СТАТЬИ (НTML-КОД):

$<$ a rel="license" href="http://creativecommons.org/licenses/by/4.0/"><img alt="Лицензия Creative Commons" style="border-width:0" src="https://i.creativecommons.org/l/by/4.0/88x31.png" / $></ \mathrm{a}><$ br $/>$ Произведение "<span xmlns:dct="http://purl.org/dc/terms/" href="http://purl.org/dc/dcmitype/Text" property="dct:title"

$\mathrm{rel}=$ "dct:type">алюминиевых сплавов редкими металлами - основа перспективных материалов в строительстве и транспорте </span>» созданное автором по имени <a xmlns:cc="http://creativecommons.org/ns\#" href="Нанотехнологии в строительстве. - 2016. - Том 8, № 3. - C. 60-69. - DOI:dx.doi.org/10.15828/2075-8545-2016-8-3-60-69."

property="cc:attributionName" rel="cc:attributionURL"> Скачков В.М., Яценко С.П. </a >, публикуется на условиях $<$ a rel="license" href="http://creativecommons.org/licenses/by/4.0/">лицензии Creative Commons «Attribution» ( Атрибуция») 4.0 Всемирная $</ \mathrm{a}>$. $<\mathrm{br} />$ Основано на произведении с $<$ a xmlns:dct="http://purl.org/dc/terms/" href="http:// nanobuild.ru/ru_RU/nanobuild-3-2016/" rel="dct:source" $>$ http://nanobuild.ru/ru_RU/nanobuild-3-2016/</a $>$. $<$ br $/>$ Pa3решения, выходящие за рамки данной лицензии, могут быть доступны на странице <a xmlns:cc="http://creativecommons. org/ns\#" href="vms@weburg.me" rel="cc:morePermissions">vms@weburg.me</a> .

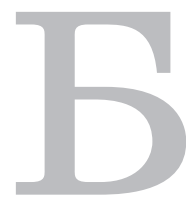

инарные диаграммы состояния алюминия с редкими металлами (РМ) со стороны алюминия преимущественно имеют перитектический тип строения с резким повышением кривой ликвидус [1]. Особенностью упрочнения сплавов с введением РМ является появление алюминидных дисперсойдов, оказывающих влияние на образование зеренной структуры в процессе последующих технологических манипуляций (прокатки, штамповки, нагрева). Дисперсойды резко повышают температуру рекристаллизации и сохраняют после термообработки субзеренное строение алюминиевых сплавов. Из редких металлов, пожалуй, наибольший интерес представляет скандий, поскольку параметры кристаллической решетки интерметаллического соединения (ИMC) $\mathrm{Al}_{3} \mathrm{Sc}$ различаются от аналогичных у решетки $\alpha$-Al всего на $1 \%$, а межфазное натяжение на границе с расплавом - в два раза меньше, чем это же значение для алюминия со своим кристаллом (сравните: 45 и 92 MH/м) [2]. Существенно улучшает механические свойства алюминиевых сплавов дополнительное введение в сплав циркония, причем суммарная концентрация скандия и циркония в получаемом сплаве должна быть около 0.3 масс.\% при соотношении $\mathrm{Zr} / \mathrm{Sc} \geq 2$ [3, 4]. Для сплава только со скандием упрочнение достигается при температуре $350^{\circ} \mathrm{C}$, a с добавкой циркония в тройной системе $\mathrm{Al}-\mathrm{Sc}-\mathrm{Zr} \geq 450^{\circ} \mathrm{C}$. $\mathrm{Paz}^{-}$ мер дисперсойдов в зависимости от содержания скандия и циркония в сплаве и температуры обжига колеблется в интервале радиуса частиц $\mathrm{Al}_{3}(\mathrm{Sc}, \mathrm{Zr})$ от 2.0 до 8.0 нм (рис. 1) [4]. Микроструктура $\mathrm{Al}_{3} \mathrm{Sc}$ в тернарных соединениях с переходными металлами IIIA (иттрий) и IVA (титан, цирконий, гафний) изучена в интервале концентраций переходного ме- 


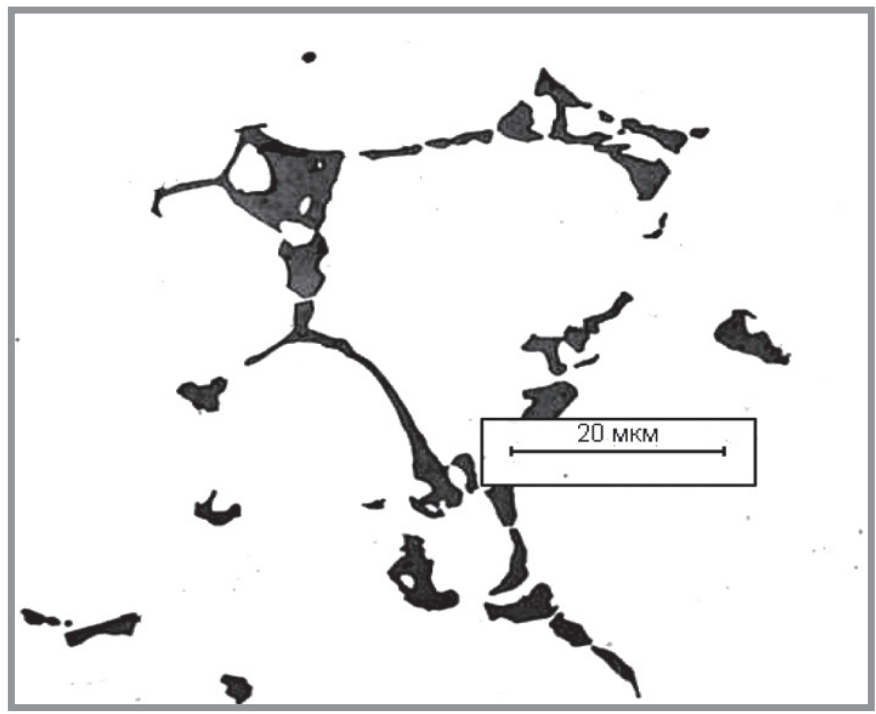

Рuc. 1. Микроструктура твёрдого раствора сплава $\mathrm{Al}-\mathrm{Mg}$ ( $\mathrm{Sc}, \mathrm{Zr}$ ).

Средний размер зерен $<40$ мкм, размер дисперсойдов 6-10 нм

талла до 0.75 ат.\% в работе [5]. В системе $\mathrm{Al}-\mathrm{Zr}-\mathrm{Y}$ тернарных соединений не обнаружено [6]. Титан и гафний также, как цирконий и иттрий, в бинарных системах с алюминием образуют соединения $\mathrm{Al}_{3} \mathrm{M}$ по перитектическим реакциям [1, 7].

Использование скандия как основного легирующего компонента повысило механические свойства $\left(\sigma_{\text {в }}\right)$ сплава АМг6 со 150 до $180 \mathrm{MПа}$ и более чем в 2 раза: для марки 1545 (Al-5.2Mg-0.3Sc-0.1Zr) - $380 \mathrm{MПа,}$ для 1970 и 1975 (систем $\mathrm{Al}-\mathrm{Zn}-\mathrm{Mg}-\mathrm{Cu}-\mathrm{Sc}$ ) - 480 и 530-550 МПа [8, 9]. Конструкционная прочность сварных изделий выше, чем у аналогичных из сплава АМг6, а коэффициент прочности сварного соединения с усиленным швом близок к 1 [8]. Такие сплавы предназначаются для различных целей. Например, изготовление и опытная проверка на нефтегазовом месторождении труб из алюминий-скандиевого сплава показала, что стоимость одной тонны труб из этого сплава и одной тонны импортных труб из нержавеющей стали примерно равны, но с учетом разницы в удельной плотности (2,9 раза) один км труб из сплава 1570 (Al-Mg-Sc) в три раза дешевле стальных. Важно, что коррозионная стойкость сплава 1570 намного выше, чем стали Х18Н10 [9].

Замена стальных вагонов на алюминиевые снижает массу вагона на $1 / 3$, увеличивает грузоподъемность, и они являются более практичными при использовании [10]. Сплавы на основе систем $\mathrm{Al}-\mathrm{Mg}-\mathrm{Li}-\mathrm{Sc}$ и $\mathrm{Al}-$ $\mathrm{Cu}-\mathrm{Li}-\mathrm{Sc}$, выпускаемые на OAO «КУМЗ», освоены в металлургическом 
производстве с поставкой листов, прессованных профилей и плит. Они рекомендованы для судостроения, конструкций фюзеляжа и крыла самолета [11]. Высокие прочностные коррозионные свойства и возможность тонколистового выпуска позволяют использовать их в слоистых полуфабрикатах типа СИАЛ [12].

Значительно повысить экономическую эффективность изготовления изделий с получением уникальных сплавов по механическим, термическим, коррозионным и радиационным свойствам позволяет использование инжекции технологического порошка в жидкий алюминиевый сплав. Авторами изучено большое число солевых систем технологических порошков на основе фторидно-хлоридных солей щелочных $(\mathrm{Li}, \mathrm{Na}, \mathrm{K})$ и щелочноземельного (Сa) металлов с введением фторидов или оксидов скандия, циркония, гафния и иттрия, для поиска оптимальных условий вытеснения их более отрицательным алюминием. Температуры плавления эвтектических составов солевых смесей меняются в зависимости от состава, от $454^{\circ} \mathrm{C}$ (для $\mathrm{LiF}-\mathrm{NaF}-\mathrm{KF}$ ) до криолита $\left(\mathrm{Na}_{3} \mathrm{AlF}_{6}\right) 965^{\circ} \mathrm{C}[13]$. Использование кальциевых солей позволяет существенно снизить содержание натрия в конечном сплаве, примесь которого существенно увеличивает объем брака при получении тонколистового проката. Для изученных солевых систем установлены значения растворимости фторида и оксида скандия в зависимости от температуры. Введение в состав технологического порошка оксида скандия несколько снижает прямой выход скандия в сплав по сравнению с фторидом скандия. Растворимость этих соединений скандия в солевых системах также существенно отличается. Например, при $800^{\circ} \mathrm{C}$ в кальциевой солевой смеси значение растворимости составляет $0.6 \mathrm{Sc}_{2} \mathrm{O}_{3}$ и в 3.4 масс.\% для $\mathrm{ScF}_{3}$. Термодинамические расчеты для реакции вытеснения скандия алюминием в лигатуру при температуре $800^{\circ} \mathrm{C}$ дают величину выхода около $80 \%$. Повышение температуры взаимодействия снижает извлечение скандия из солевого расплава, а ведение процесса при температуре около $700^{\circ} \mathrm{C}$ позволяет иметь прямой выход скандия в сплав более $95 \%$. Инжекция порошка инертным газом в алюминий, защищенного покрывным флюсом от окисления, способствует удалению ряда примесей из сплава. При использовании исходного первичного алюминия марки А85, химический состав примесей сплава (исходный - числитель, после плавки - знаменатель, масс.\% ) определен равным: Cu 0.01/0.0019; Mg 0.01/0.0007; Zn 0.01/0.01; Sc 0.000/0.78; 
Mn 0.01/0.003; Si 0.05/0.038; Na 0.0014/0.0012. Водорода в сплаве $<0.29 \mathrm{~m}^{3} / 100$ г. В лабораторных условиях очистка от примесей достигалась при несколько лучших значениях. Особенно это касалось железа, которое в промышленной печи попадало от предшествующих штатных операций плавки и по анализам не очищалось от содержания в исходном алюминии. Модифицирование алюминиевых сплавов скандием на ОАО «КУМЗ» проводили в индукционной печи при температуре 780$800^{\circ} \mathrm{C}$, для присадки порошка в расплавы использовалась инжекционная установка УФР-20 (рис. 2).

После завершения операции инжекции аргоном и небольшой выдержки шлак снимали при температуре $730^{\circ} \mathrm{C}$. При этой же температуре проводили литье в водоохлаждаемые изложницы. Проводились особые плавки, где в качестве базового порошка использовалась смесь из хлоридно-фторидных кальциевых солей с соответствующими добавками $\left(\mathrm{AlF}_{3}, \mathrm{ScF}_{3}\right.$ и $\left.\mathrm{KHF}_{2}\right)$, при этом в полученных алюминиевых сплавах содержание натрия было установлено ниже предельно допустимой концентрации и составляло в сплаве $1545 \mathrm{~K}(60-66) \cdot 10^{-5}$, а кальция в нем было меньше 0.01 масс.\% . При плавках солевой смесью $\mathrm{KCl}, \mathrm{AlF}_{3}, \mathrm{ScF}_{3}$, $\mathrm{KHF}_{2}$ и с введением в состав шихты алюминия марки А85, магния Мг90 и лигатур алюминиевых с $\mathrm{Zr}, \mathrm{Be}, \mathrm{Cr}, \mathrm{Mn}$, и $\mathrm{Ti}$, приготовленных на чушковом алюминии марки А85, содержание натрия в слитках составляло $(53-95) \cdot 10^{-5}$ мacc. $\%$.
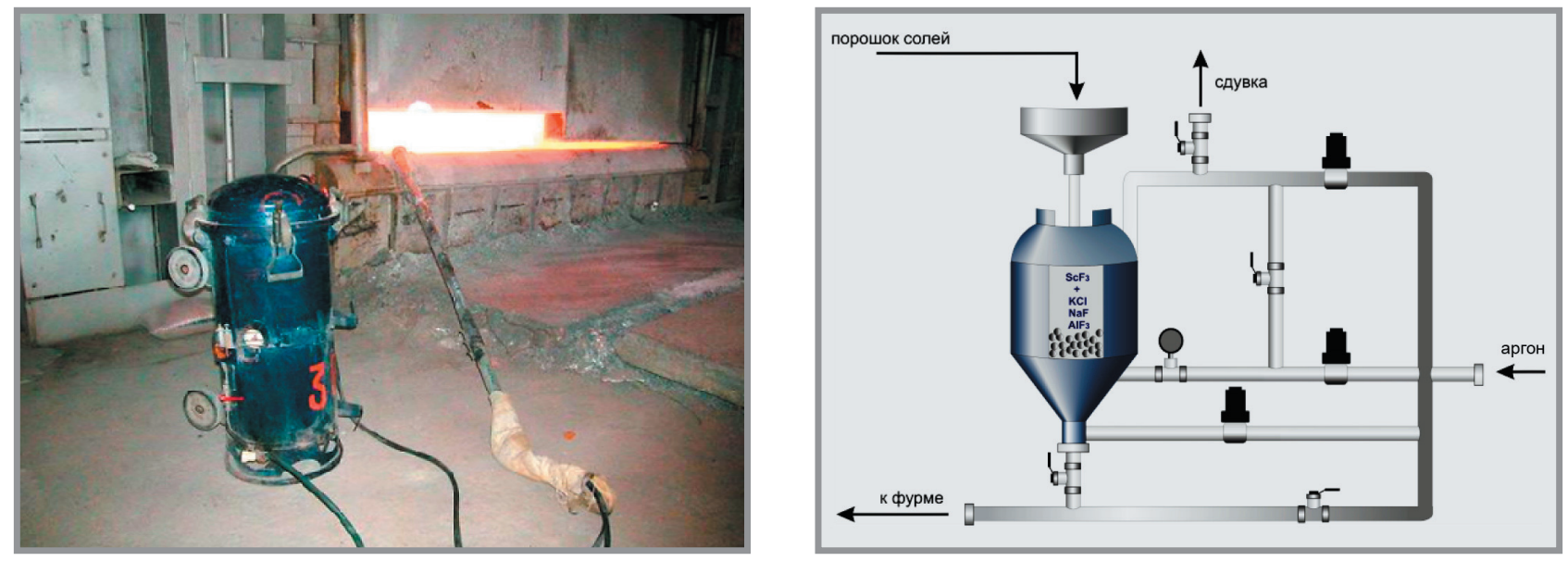

Puc. 2. Применение инжекционной технологии получения алюминиевых сплавов, содержащих скандий, и схема питателя технологического порошка на ОАО «КУМЗ» 


\section{Заключение}

Инжекционный метод получения сплавов позволяет вводить в технологический порошок необходимое количество других ингредиентов в виде фторидных или оксидных соединений, металлы которых являются более положительными по электрохимической шкале. В лабораторных условиях это нами успешно подтверждено [14]. Инжекционный способ позволяет упростить технологию получения заданных сплавов.

В строительной индустрии, так же, как и в транспортной сфере, включая самолето- и ракетостроение, применение новых легких, более прочных и коррозионно-устойчивых алюминиевых сплавов экономно и позволит развивать высокие технологии и удешевлять производство [15]. Перспективы получения в нужных масштабах РМ в России существуют не только на основе новых богатых этими металлами месторождениях, как например Томторское, но и в шламовых полях цветной и черной металлургии.

УВАЖАЕМЫЕ КОЛЛЕГИ!

ПРИ ИСПОЛЬЗОВАНИИ МАТЕРИАЛА ДАННОЙ СТАТЬИ

ПРОСИМ ДЕЛАТЬ БИБЛИОГРАФИЧЕСКУЮ ССЫЛКУ НА НЕЁ:

Скачков В.М., Яценко С.П. Модифицирование алюминиевых сплавов редкими металлами - основа перспективных материалов в строительстве и транспорте // Нанотехнологии в строительстве. - 2016. - Том 8, № 3. - С. 60-69. - DOI: dx.doi.org/10.15828/2075-8545-2016-8-3-60-69.

\section{DeAR COLLEAGUES!}

THE REFERENCE TO THIS PAPER HAS THE FOLLOWING CITATION FORMAT:

Skachkov V.M., Yatsenko S.P. Modification of aluminium alloys with rare metals the basis for advanced materials in construction and transport. Nanotehnologii $\mathrm{v}$ stroitel'stve = Nanotechnologies in Construction. 2016, Vol. 8, no. 3, pp. 60-69. DOI: dx.doi.org/10.15828/2075-8545-2016-8-3-60-69. (In Russian). 


\section{Библиографический список:}

1. Мондольро Л.Ф. Структура и свойства алюминиевых сплавов. - М.: Металлургия. $-1979 .-640$ с.

2. Яиенко С.П., Пасечник Л.А., Скачков В.М. Скандий: получение и применение // Наука и техника. - 2015. - №3 (15). - С. 32-37.

3. Optimization of Hardening of Al-Zr-Sc cast alloys / N.A. Belov, A.N. Alabin, D.G. Eskin et al. // J. Mater Sci. - 2006. - N 41. - P. 5890-5899.

4. Fuller Ch.B., Seidnan D.N., Dunand D.C. Mechanical properties of Al (Sc,Zr) alloys of ambient and elevated temperatures // Acta Materialia. - 2003. - V. 51. P. 4803-4814.

5. Harada Y., Dunand D.C. Microstructure of Al3Sc witu ternary transition-metal additions // Materials Scien. a. Engineering. - 2002. - V. A. 329-331. - P. 686695.

6. Experimental Study of Al-Zr-Y system phase equilbria of $773 \mathrm{~K} /$ She. Jia, Y. Zman, Zh. Hu a. al. // J. of Alloys and Compounds. - 2010. - V. 497. - P. 118-120.

7. Исследование богатой алюминием части диаграммы состояния $\mathrm{Al}-\mathrm{Hf} /$ Л.Л. Рохлин, Н.Р. Богвар, Т.В. Добаткина и др. // Металлы. - 2009. - № 3. C. 93-98.

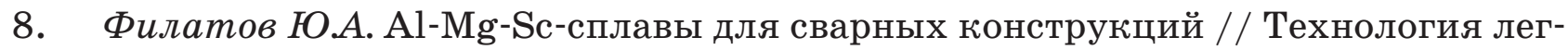
ких сплавов. - 2004. - № 5. - С. 13-19.

9. Перспективы извлечения скандия из растворов подземного выщелачивания минерального сырья / В.П. Казанцев, Л.Р. Бекетов, Ю.П. Кудрявский и др. // Цветная металлургия. - 2009. - № 1. - С. 37-41.

10. Чертовиков В. Проблема в том, что возим не пассажиров, а тару // Металлы Евразии. - 2007. - № 2. - С. 28-29.

11. Хохлова Л.Б., Колобнев Н.И., Михайлов Е.Д. Алюминийлитиевые сплавы для самолётостроения // Металлург. - 2012. - № 5. - С. 31-35.

12. Овсянников Б.В. Новый алюминиево-литиевый сплав системы $\mathrm{Al}-\mathrm{Cu}-\mathrm{Mg}-$ $\mathrm{Li}-(\mathrm{Ag}, \mathrm{Sc})$, предназначенный для изготовления тонких листов и профилей // Цветные металлы. - 2014. - № 11. - С. 90-94.

13. Получение алюминий-скандиевых сплавов методом инжекции технологических порошков в расплав / Б.В. Овсянников, С.П. Яценко, П.А. Варченя и др. // Технология металлов. - 2011. - № 5. - С. 23-29.

14. Скачков B.M., Яиенко С.П. Получение Sc-, Zr-, Hf-, Y-лигатур на основе алюминия методом высокотемпературных обменных реакций в расплавах солей // Цветные металлы. - 2013. - № 12. - С. 48-52.

15. Каблов E.Н. Без новых материалов - нет будущего // Металлург. - 2013. № 12. - С. 4-8. 